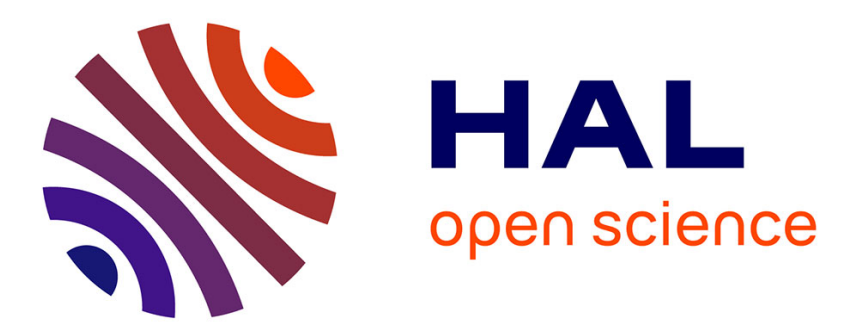

\title{
3D acoustic-(visco)elastic coupled formulation and its spectral-element implementation on a Cartesian-based hexahedral mesh
}

Jian Cao, Romain Brossier, Ludovic Métivier

\section{- To cite this version:}

Jian Cao, Romain Brossier, Ludovic Métivier. 3D acoustic-(visco)elastic coupled formulation and its spectral-element implementation on a Cartesian-based hexahedral mesh. SEG Technical Program, Oct 2020, Houston, United States. hal-03099621

\section{HAL Id: hal-03099621 \\ https://hal.science/hal-03099621}

Submitted on 6 Jan 2021

HAL is a multi-disciplinary open access archive for the deposit and dissemination of scientific research documents, whether they are published or not. The documents may come from teaching and research institutions in France or abroad, or from public or private research centers.
L'archive ouverte pluridisciplinaire HAL, est destinée au dépôt et à la diffusion de documents scientifiques de niveau recherche, publiés ou non, émanant des établissements d'enseignement et de recherche français ou étrangers, des laboratoires publics ou privés. 


\section{D acoustic-(visco)elastic coupled formulation and its spectral-element implementation on a Cartesian-based hexahedral mesh \\ Jian Cao $^{1 *}$, Romain Brossier ${ }^{1}$, Ludovic Métivier ${ }^{1,2}$}

1 Univ. Grenoble Alpes, ISTerre, F-38058 Grenoble, France

2 Univ. Grenoble Alpes, CNRS, LJK, F-38058 Grenoble, France

\section{SUMMARY}

Ocean Bottom Node (OBN) acquisition is becoming popular in the exploration of challenging marine environments. A major advantage over conventional streamer acquisition is its ability of capturing converted wave by recording both $\mathrm{P}$ - and S-wave on the solid seabed. Thus, to study and process OBN data, modeling of seismic wave propagation in fluid-solid coupled media needs to be taken into account. In this study, we apply a partitioned approach to fluid-solid coupled media. Fluid and solid domains are divided explicitly and handled with the acoustic-wave and (visco)elastic-wave equation, respectively. The mutual interaction between these two wave-equations is modeled by boundary conditions at the fluid-solid interface. This leads to a coupled acoustic-(visco)elastic wave-equation system. According to the wavefield variables used in the acoustic-wave equation, we compare 4 acoustic-(visco)elastic coupled formulations in terms of pressure, velocity potential, displacement potential and displacement, respectively. The spectral-element method (SEM) is used as a numerical modeling tool to reveal their pros and cons from the aspects of complexity, accuracy and computational efficiency. Finally, we present various fluid-solid coupled modeling examples including isotropic elastic, anisotropic elastic (VTI and TTI) and anelastic media on the Cartesian-based hexahedral mesh. They are all implemented with the displacement potential formulation which achieves the best trade-off compared with the other three.

\section{INTRODUCTION}

As the marine seismic exploration moves to complex deep-water geologic environments, Ocean Bottom Node (OBN) acquisition begins to be used as an effective technology for imaging quality enhancement and risk reduction. Compared with the conventional streamer acquisition, it has the advantages of decoupling the source from the receivers, providing wide-azimuth illumination and capturing converted wave by recording both P- and S-wave on the solid seabed (Maver 2011).

For the purpose of studying and processing OBN data, an accurate modeling of seismic wave propagation would require to be implemented in fluid-solid coupled media to produce elastic effects like P to $\mathrm{S}$ conversions. Typically, there are two approaches to model the seismic wave propagation in fluid-solid coupled media: monolithic and partitioned approaches (Hou et al. 2012, De Basabe and Sen 2015). The monolithic approach uses the same governing equation in both fluid and solid domains, and the fluid-solid interface is tackled implicitly. However, this approach is not accurate enough due to dispersion and discretization errors near the interface (De Basabe and Sen 2015). In addition, as pointed out by Komatitsch et al. (2000), the monolithic approach based on the second-order displacement formulation of the elastic-wave equation yields strong artifacts in the fluid region when using a conventional spectral-element (SEM) method for the discretization.

To avoid those shortcomings, we consider the partitioned approach to perform simulations in fluid-solid coupled media. The medium is divided into solid and fluid domains. The acoustic-wave equation is used to describe the wave propagation in the fluid domain, while the (visco)elastic-wave equation models elastic vibrations in the solid domain. To account for the mutual interaction between the two domains, fluid-solid boundary conditions are used to connect the two waveequations, leading to a coupled acoustic-(visco)elastic wave-equation system. In the solid domain, the (visco)elastic-wave equation is commonly formulated in terms of displacement. The fluid, however, can be modeled in terms of pressure, velocity potential, displacement poten-

tial or displacement (Everstine 1997), yielding 4 acoustic-(visco)elastic coupled formulations (Feng | 2000, Komatitsch et al. 2000. Chaljub and Valette 2004, Ross et al. 2009. During the implementation, the SEM is used for the spatial discretization for its high accuracy in relatively smooth media, interface conforming and high computational efficiency through domain-decomposition parallelization (Komatitsch 1997. Peter et al. 2011).

In the following sections, we first conduct a comparison study between the 4 acoustic-(visco)elastic coupled formulations. We show how these coupling formulations can be expressed within the SEM framework. Then, for simplicity, several benchmark tests in 2D are carried out to reveal their pros and cons in terms of accuracy and computational efficiency. We conclude that the best trade-off is achieved by the displacement potential formulation. Finally, we consider a 3D parallel implementation of this formulation and present various modeling examples including isotropic elastic, anisotropic elastic (VTI and TTI) and anelastic media on the Cartesian-based hexahedral mesh, in the perspective to extend its application to marine seismic imaging.

\section{FORMULATE THE ACOUSTIC-(VISCO)ELASTIC COUPLED} PROBLEM

In the solid region, the complete form of the viscoelastic-wave equation can be written as

$$
\rho_{s} \ddot{\mathbf{u}}_{s}=\nabla \cdot \boldsymbol{\sigma}+\mathbf{f}_{s}, \quad \boldsymbol{\sigma}=\mathbf{M} *_{t} \boldsymbol{\varepsilon}, \quad \boldsymbol{\varepsilon}=\frac{1}{2}\left[\nabla \mathbf{u}_{s}+\left(\nabla \mathbf{u}_{s}\right)^{T}\right],
$$

where $\mathbf{u}_{s}$ and $\mathbf{f}_{s}$ are the displacement and force vectors, respectively, $\boldsymbol{\sigma}$ is the stress tensor, $\boldsymbol{\varepsilon}$ is the strain tensor, $\rho_{s}$ is the solid density, and the attenuation effect is described by the relaxation rate $\mathbf{M}$ and time convolution $*_{t}$. For the pure elastic-wave equation, the relaxation rate is

$$
\mathbf{M}=\mathbf{C} \delta(t),
$$

where $\mathbf{C}$ is the elastic stiffness coefficient and $\delta(t)$ is the Dirac delta function.

Assuming an irrotational and inviscid fluid, its wavefield is governed by the following conservation and dynamic equations:

$$
\rho_{f} \ddot{\mathbf{u}}_{f}+\nabla P=\mathbf{f}_{f}, \quad \dot{P}+\kappa \nabla \cdot \dot{\mathbf{u}}_{f}=0,
$$

where $\mathbf{u}_{f}$ and $\mathbf{f}_{f}$ are the displacement and force vectors in the fluid domain, respectively, $P$ is the pressure, $\rho_{f}$ is the fluid density, and $\kappa$ is the bulk modulus of the fluid. By substitution and elimination, Eq. (3) can be rewritten in terms of fluid pressure $(P)$, displacement potential $(\varphi)$, velocity potential $(\phi)$ or displacement $\left(\mathbf{u}_{f}\right)$, respectively:

$$
\begin{gathered}
\ddot{P}+\kappa \nabla \cdot\left(-\frac{1}{\rho_{f}} \nabla P\right)=P_{f}, \quad \ddot{\varphi}-\kappa \nabla \cdot\left(\frac{1}{\rho_{f}} \nabla \varphi\right)=\iint-P_{f} d t d t, \\
\ddot{\phi}-\frac{\kappa}{\rho_{f}} \nabla \cdot \nabla \phi=\int-\frac{1}{\rho_{f}} P_{f} d t, \quad \rho_{f} \ddot{\mathbf{u}}_{f}-\nabla\left(\kappa \nabla \cdot \mathbf{u}_{f}\right)=\mathbf{f}_{f} .
\end{gathered}
$$

Here, $P_{f}$ is the pressure source related to the force vector $\left(\mathbf{f}_{f}\right)$, and the velocity potential $(\phi)$ and displacement potential $(\varphi)$ are defined by

$$
\dot{\mathbf{u}}_{f}:=\nabla \phi, \quad \mathbf{u}_{f}:=\frac{1}{\rho} \nabla \varphi .
$$

Their relationships with pressure $(P)$ follow

$$
P=-\rho \dot{\phi}, \quad P=-\ddot{\varphi} .
$$

Accordingly, the coupling conditions for the connection of the acousticwave and (visco)elastic-wave equation can be written in 4 different 


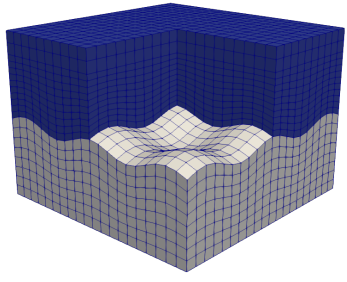

(a)

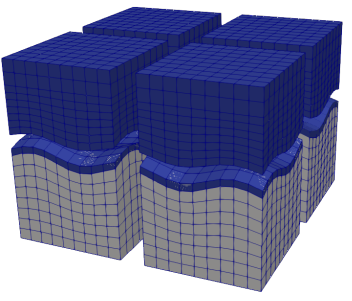

(b)
Figure 1: Cartesian-based hexahedral mesh for fluid-solid coupled modeling (a) and partitions on 8 cores (b). Here the blue parts indicate the fluid domain.

ways at the fluid-solid interface:

1) $P-\mathbf{u}_{s}$ formulation

$$
\ddot{\mathbf{u}}_{s} \cdot \mathbf{n}_{s}=\frac{1}{\rho_{f}} \nabla P \cdot \mathbf{n}_{f}, \quad \boldsymbol{\sigma}_{s} \cdot \mathbf{n}_{s}=P \mathbf{n}_{f},
$$

2) $\varphi-\mathbf{u}_{s}$ formulation

$$
\mathbf{u}_{s} \cdot \mathbf{n}_{s}=-\frac{1}{\rho_{f}} \nabla \varphi \cdot \mathbf{n}_{f}, \quad \boldsymbol{\sigma}_{s} \cdot \mathbf{n}_{s}=-\ddot{\varphi} \mathbf{n}_{f},
$$

3) $\phi-\mathbf{u}_{s}$ formulation

$$
\dot{\mathbf{u}}_{s} \cdot \mathbf{n}_{s}=-\nabla \phi \cdot \mathbf{n}_{f}, \quad \boldsymbol{\sigma}_{s} \cdot \mathbf{n}_{s}=-\rho_{f} \dot{\phi} \mathbf{n}_{f},
$$

4) $\mathbf{u}_{f}-\mathbf{u}_{s}$ formulation

$$
\mathbf{u}_{s} \cdot \mathbf{n}_{s}=-\mathbf{u}_{f} \cdot \mathbf{n}_{f}, \quad \boldsymbol{\sigma}_{s} \cdot \mathbf{n}_{s}=-\kappa\left(\nabla \cdot \mathbf{u}_{f}\right) \mathbf{n}_{f} .
$$

Note that the continuity of normal component of displacement $(\mathbf{u} \cdot \mathbf{n})$ is not strictly satisfied in cases 1) $P-\mathbf{u}_{s}$ and 3) $\phi-\mathbf{u}_{s}$, where only the normal component of acceleration $\ddot{\mathbf{u}}$ and velocity $\dot{\mathbf{u}}$ are enforced to be continuous, respectively. We show how this affects the SEM results in the numerical tests.

\section{DISCRETIZATION USING SEM}

When using the SEM, we need to rewrite the (visco)elastic-wave and acoustic-wave equations (Eqs. (1) and (4) in the weak form by dotting with arbitrary test functions, and the above coupling conditions need to be met in weak forms. Consequently, we obtain 4 semi-discretized equation systems for representing the fluid-solid coupled modeling. For brevity, we write these systems in matrix form and categorize them according to their symmetry. The following is the case of the acousticelastic coupled system:

1) $P-\mathbf{u}_{s}$ system (non-symmetric)

$\left(\begin{array}{cc}M_{f} & \mathbf{A} \\ \mathbf{0} & \mathbf{M}_{s}\end{array}\right)\left(\begin{array}{c}\ddot{P} \\ \ddot{\mathbf{u}}_{s}\end{array}\right)+\left(\begin{array}{cc}D_{f} & \mathbf{0} \\ \mathbf{0} & \mathbf{D}_{s}\end{array}\right)\left(\begin{array}{c}\dot{P} \\ \dot{\mathbf{u}}_{s}\end{array}\right)+\left(\begin{array}{cc}K_{f} & \mathbf{0} \\ \mathbf{A}^{\mathbf{T}} & \mathbf{K}_{s}\end{array}\right)\left(\begin{array}{c}P \\ \mathbf{u}_{s}\end{array}\right)=\left(\begin{array}{c}P_{f} \\ \mathbf{f}_{s}\end{array}\right)$,

2) $\varphi-\mathbf{u}_{s}$ system (non-symmetric)

$\left(\begin{array}{cc}M_{f} & \mathbf{0} \\ \mathbf{A}^{\mathbf{T}} & \mathbf{M}_{s}\end{array}\right)\left(\begin{array}{c}\ddot{\varphi} \\ \ddot{\mathbf{u}}_{s}\end{array}\right)+\left(\begin{array}{cc}D_{f} & \mathbf{0} \\ \mathbf{0} & \mathbf{D}_{s}\end{array}\right)\left(\begin{array}{c}\dot{\varphi} \\ \dot{\mathbf{u}}_{s}\end{array}\right)+\left(\begin{array}{cc}K_{f} & \mathbf{A} \\ \mathbf{0} & \mathbf{K}_{s}\end{array}\right)\left(\begin{array}{c}\varphi \\ \mathbf{u}_{s}\end{array}\right)=\left(\begin{array}{c}\varphi_{f} \\ \mathbf{f}_{s}\end{array}\right)$,

3) $\phi-\mathbf{u}_{s}$ system (symmetric)

$\left(\begin{array}{cc}M_{f} & \mathbf{0} \\ \mathbf{0} & \mathbf{M}_{s}\end{array}\right)\left(\begin{array}{c}\ddot{\phi} \\ \ddot{\mathbf{u}}_{s}\end{array}\right)+\left(\begin{array}{cc}D_{f} & \mathbf{A} \\ \mathbf{A}^{\mathbf{T}} & \mathbf{D}_{s}\end{array}\right)\left(\begin{array}{c}\dot{\phi} \\ \dot{\mathbf{u}}_{s}\end{array}\right)+\left(\begin{array}{cc}K_{f} & \mathbf{0} \\ \mathbf{0} & \mathbf{K}_{s}\end{array}\right)\left(\begin{array}{c}\phi \\ \mathbf{u}_{s}\end{array}\right)=\left(\begin{array}{c}\phi_{f} \\ \mathbf{f}_{s}\end{array}\right)$, (13)

4) $\mathbf{u}_{f}-\mathbf{u}_{s}$ system (symmetric)

$$
\left(\begin{array}{cc}
\mathbf{M}_{f} & \mathbf{0} \\
\mathbf{0} & \mathbf{M}_{s}
\end{array}\right)\left(\begin{array}{c}
\ddot{\mathbf{u}}_{f} \\
\ddot{\mathbf{u}}_{s}
\end{array}\right)+\left(\begin{array}{cc}
\mathbf{D}_{f} & \mathbf{0} \\
\mathbf{0} & \mathbf{D}_{s}
\end{array}\right)\left(\begin{array}{c}
\dot{\mathbf{u}}_{f} \\
\dot{\mathbf{u}}_{s}
\end{array}\right)+\left(\begin{array}{cc}
\mathbf{K}_{f} & \mathbf{A} \\
\mathbf{A}^{\mathrm{T}} & \mathbf{K}_{s}
\end{array}\right)\left(\begin{array}{c}
\mathbf{u}_{f} \\
\mathbf{u}_{s}
\end{array}\right)=\left(\begin{array}{c}
\mathbf{f}_{f} \\
\mathbf{f}_{s}
\end{array}\right)
$$

Here, $\mathbf{A}$ is the coupling matrix obtained by the integration along the

\begin{tabular}{|c|c|c|c|c|c|}
\hline$\overline{\text { Media }}$ & Property & rho $(\mathrm{kg} / \mathrm{m}$ & $T_{p}(\mathrm{~m} /$ & $V_{s}(\mathrm{~m} / \mathrm{s})$ & Elastic \\
\hline Fluid & Acoustic & 1000 & 1500 & 0 & Parameters \\
\hline \multirow{4}{*}{ Solid } & E-ISO & \multirow{4}{*}{2500} & \multirow{4}{*}{3400} & \multirow{4}{*}{1963} & N/A \\
\hline & E-VTI & & & & $\overline{\varepsilon=0.1, \delta=0.4, \gamma=0.2}$ \\
\hline & E-TTI & & & & $\varepsilon=0.1, \delta=0.4, \gamma=0.2$ \\
\hline & $\overline{\text { E-VISCO }}$ & & & & $\begin{array}{l}\theta=45^{\circ}, \phi=15^{\circ} \\
O_{n}=40, O_{c}=30\end{array}$ \\
\hline
\end{tabular}
fluid-solid interfaces, and the remaining matrices correspond to conventional notations in SEM, namely, $M_{f}$ and $\mathbf{M}_{s}$ are the mass matrices, $D_{f}$ and $\mathbf{D}_{s}$ are the matrices for implementing absorbing boundary condition, and $K_{d}$ and $\mathbf{K}_{s}$ are the stiffness matrices. The wavefields for
Table 1: Physical parameters of the bilayered model for numerical tests.

both fluid and solid domains are represented locally by the piecewisepolynomial approximation within the reference element $(\varepsilon, \eta, \zeta)$

$$
\begin{aligned}
& P(\varepsilon, \eta, \zeta)=\sum_{i=0}^{N} \sum_{j=0}^{N} \sum_{k=0}^{N} P_{i, j, k} \iota_{i}(\varepsilon) \iota_{j}(\eta) \iota_{k}(\zeta), \\
& \varphi(\varepsilon, \eta, \zeta)=\sum_{i=0}^{N} \sum_{j=0}^{N} \sum_{k=0}^{N} \varphi_{i, j, k} \iota_{i}(\varepsilon) \iota_{j}(\eta) \iota_{k}(\zeta), \\
& \phi(\varepsilon, \eta, \zeta)=\sum_{i=0}^{N} \sum_{j=0}^{N} \sum_{k=0}^{N} \phi_{i, j, k} \iota_{i}(\varepsilon) \iota_{j}(\eta) \iota_{k}(\zeta),
\end{aligned}
$$

and

$$
\mathbf{u}(\varepsilon, \eta, \zeta)=\sum_{i=0}^{N} \sum_{j=0}^{N} \sum_{k=0}^{N} \mathbf{u}_{i, j, k} \boldsymbol{\iota}_{i}(\varepsilon) \boldsymbol{\iota}_{j}(\eta) \boldsymbol{\iota}_{k}(\zeta)
$$

respectively, where $\varepsilon \in[-1,1], \eta \in[-1,1], \zeta \in[-1,1] . \imath_{i}(\varepsilon), \iota_{j}(\eta)$ and $l_{k}(\zeta)$ are $1 \mathrm{D}$ Lagrange interpolants in the $\varepsilon$-, $\eta$ - and $\zeta$-directions, respectively.

Note that when considering the attenuation contribution in the solid domain, an extra term $\left[0,-\mathbf{F}\left(\boldsymbol{\psi}_{v}\right)\right]^{T}$ needs to be added on the left hand side of each system, where $\boldsymbol{\psi}_{v}$ is the memory variables satisfying a specific first-order ordinary differential equation (Yang et al. 2016).

\section{NUMERICAL TESTS}

As shown above, the $\mathbf{u}_{\mathbf{f}}-\mathbf{u}_{\mathbf{s}}$ system uses a vector-valued wave equation in the fluid domain. Therefore, it is less efficient than the other three using a scalar-valued equation in the fluid domain for seismic modeling. For this reason, we focus on the three systems where a scalar-valued equation is considered in the fluid domain. The computational mesh is a Cartesian-based hexahedral mesh (Fig. 17), which combines the flexibility of interface conforming and the convenience of partition in the domain-decomposition based parallelization (Fig. 1p).

\section{Validation of numerical solutions}

For simplicity, the validation tests are carried out in 2D. SEM results are benchmarked against the $2 \mathrm{D}$ semi-analytical solution of a bilayered model (Diaz and Ezziani, 2008). The physical parameters of this model are listed in Table 1 The upper part is acoustic, while the lower part is isotropic pure elastic. The Lagrange polynomial degree we use is $N=5$. A total number of $520 \times 200=104000$ elements is used. An explosive pressure-source with a $10 \mathrm{~Hz}$ Ricker wavelet is applied $0.5 \mathrm{~km}$ above the interface. A combination of sponge layers (Cerjan et al. 1985) and radiative boundary condition (Lysmer and Kuhlemeyer 1969) is used on all the edges of the model to attenuate outgoing waves.

Figure 2 shows the spectra of source-time functions for systems of $P-\mathbf{u}_{s}, \phi-\mathbf{u}_{s}$ and $\varphi-\mathbf{u}_{s}$, where wavelets of $\phi$ and $\varphi$ are obtained by single and double time-integration of the pressure wavelet, respectively (Eq. (4)). The dominant frequencies for $\phi$ and $\varphi$ wavelet both shift towards low frequency. This means an increase of low-frequency component for both source-time functions, and Figure 2 displays that the attenuation effectiveness of absorbing boundaries for wavefield energy decreases in these two systems. However, the physical variable of interest in the fluid domain is pressure. As Eq. 6 shows, it can be obtained through first- or second-order derivation of potential variables, respectively, and we observe a similar attenuation effectiveness 


\section{D acoustic-(visco)elastic coupled modeling}

\begin{tabular}{lcc}
\hline & Elapsed time (s) & Memory (MB) \\
\hline$P-\mathbf{u}_{s}$ system & 316.78 & 235.62 \\
\hline$\phi-\mathbf{u}_{s}$ system & 445.77 & 235.67 \\
\hline$\varphi-\mathbf{u}_{s}$ system & 319.77 & 235.62 \\
\hline
\end{tabular}

Table 2: Comparison of elapsed-time and memory consumption for $P-\mathbf{u}_{s}, \phi-\mathbf{u}_{s}$ and $\varphi-\mathbf{u}_{s}$ systems.

for the pressure using the different systems (Fig 24). Analogously, an attenuation comparison of wavefield energy in the solid domain is performed in Figure 21 to further demonstrate the absorbing consistency of the different systems. Thus, a similar numerical accuracy for these three coupling systems is achieved, when comparing the seismograms recorded on the seabed with an offset of $12.6 \mathrm{~km}$ (Fig. 3 $)$.

However, if a continuity check of displacement components is conducted at the fluid-solid interface (Fig. 3p), we find that only the $\varphi-\mathbf{u}_{s}$ system produces the same vertical displacement component from both fluid and solid sides. This is because the other two systems enforce the normal component of acceleration and velocity to be continuous respectively. Therefore, from the aspect of accuracy, $\varphi-\mathbf{u}_{s}$ system is a better choice for fluid-solid coupled modeling.

In addition, the comparison in terms of elapsed time and memory consumption in Table 2 reveals a higher computational efficiency of $P-\mathbf{u}_{s}$ and $\varphi-\mathbf{u}_{s}$ systems. The reason is that with non-symmetric coupling formulations $\left(P-\mathbf{u}_{s}\right.$ and $\left.\varphi-\mathbf{u}_{s}\right)$, the acoustic- and (visco)elastic-wave equations can be solved separately by explicit time-stepping schemes. However, the symmetric system $\phi-\mathbf{u}_{s}$ is implicit and needs to solve equations governing the fluid and solid domain simultaneously (Mönkölä 2011). Here we use a staggered prediction/multicorrection iterative scheme (Antonietti et al. 2019. Komatitsch et al. 2000) to solve the $\phi-\mathbf{u}_{s}$ system, and it requires at least two iterations at each time step.

\section{D parallel implementation and load balancing}

In this section, we discuss the 3D parallel implementation of fluidsolid coupled modeling based on the domain-decomposition strategy. We use the $\varphi-\mathbf{u}_{s}$ formulation, which achieves the best trade-off in terms of accuracy and computational efficiency following the above $2 \mathrm{D}$ tests. This implementation is developed in the framework of the SEISCOPE SEM46 code (Brossier and Trinh 2017, Trinh et al. 2019), which is a time-domain (visco)elastic wave-equation modeling and full waveform inversion package. For the implementation, the fluid free-surface boundary condition and the fluid-solid boundary condition are both taken into consideration. Figure 4 shows modeling results of seismograms (Fig 4 p) and snapshots (Fig 4 ) in the isotropic, viscous-isotropic, VTI and TTI cases, respectively, using the physical parameters listed in Table 1 The source is located in the middle of $x-y$ plane at $1 \mathrm{~km}$ depth below the sea level, and the receiver line is located on the seabed along the $x$-direction with a constant $y$-offset of $0.1 \mathrm{~km}$. As a validation, we choose a single trace whose $x$-offset is $2.8 \mathrm{~km}$, and compare its 4-component $\mathrm{OBN}$ data with results produced by SPECFEM3D (Peter et al. 2011) in the isotropic case. A good agreement can be observed in Figure 4 .

For the parallel high performance computing (HPC) aspect of the SEM46 code, the 3D Cartesian-based hexahedral mesh of SEM46 is split into regular cubic sub-domains using a conventional domain-decomposition strategy. Its parallel load balancing in elastic modeling is achieved by approaching the same number of elements (same size of sub-mesh) for each domain in the decomposition, reaching usually a parallel efficiency close to $90-95 \%$. When considering fluid-solid coupled modeling, however, the different wave-equations involved in each part of the mesh lead to load imbalance if the same decomposition strategy is used. Figure 5 a shows an example of parallel efficiency for the full acoustic modeling, the full elastic modeling and newly developed fluid-solid coupled version of SEM46 without any load-balancing optimization (without LB). In such a case, the acoustic domains have less computation to perform, leading to the waiting for elastic computation to be finished, and the overall "time-to-solution" is driven by the elastic domains. Evaluating theoretically and practically, the computation amount for elastic and acoustic elements shows a ratio of 3 between both. Using this ratio, we optimized the domain-decomposition strat-

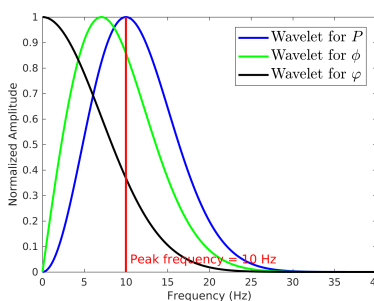

(a)

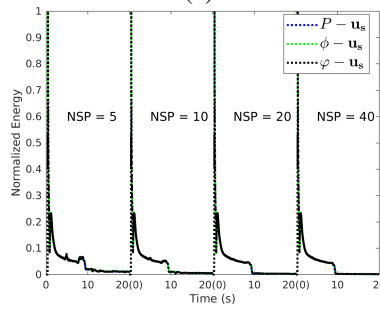

(c)

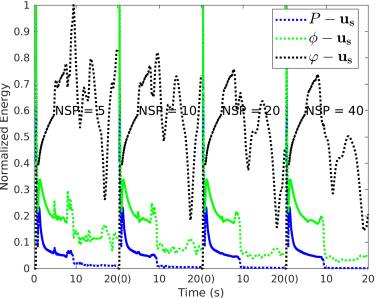

(b)

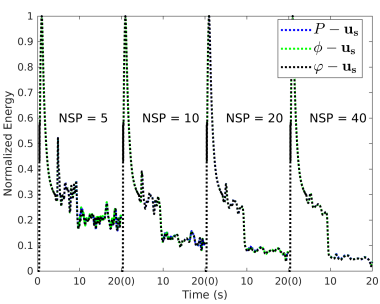

(d)
Figure 2: Evaluation of absorbing effectiveness for artificial boundary in $P-\mathbf{u}_{s}, \phi-\mathbf{u}_{s}$ and $\varphi-\mathbf{u}_{s}$ systems. (a) Spectra of source-time function, (b) absorbing effectiveness of wavefield variables $(P, \phi$ and $\varphi)$ used in the acoustic-wave equation, (c) absorbing effectiveness of pressure in the fluid domain, (d) absorbing effectiveness of displacement in the solid domain. NSP: number of sponge elements.

egy by weighting each element in the decomposition, proportionally to the amount of expected computation. This leads to an optimized domain decomposition with more acoustic elements in sub-domains, allowing to improve the parallel load-balancing significantly, as shown in Figure 5a with the "with LB" curves. In addition, our implementation considers an additional constraint for the mesh partitioning in order to avoid the fluid-solid boundary to match a domain frontier: considering this constraint makes possible to avoid any additional MPI communication for the coupling problem.

For completeness, we present the efficiency comparison of fluid-solid coupled modeling in the isotropic, viscous-isotropic, VTI and TTI cases in Figure 5 p. It also shows a good scalability in each case.

\section{CONCLUSION}

We have investigated 4 acoustic-(visco)elastic coupled formulations for modeling seismic wave propagation in fluid-solid coupled media using the spectral element method. Their corresponding semi-discretized equation systems are established and compared. Numerical tests in $2 \mathrm{D}$ indicate that the $\varphi-\mathbf{u}_{s}$ system has a better performance in terms of accuracy and computational efficiency than the other three, and the double integration of source-time function for the displacement potential $(\varphi)$ does not decrease the attenuation effectiveness of artificial boundary in modeling the physical wavefields of interest. In the 3D parallel implementation, we present various fluid-solid coupled modeling examples including isotropic elastic, anisotropic elastic (VTI and TTI) and anelastic media on the Cartesian-based hexahedral mesh and use a cost-dependent mesh partitioning to achieve the load balancing. It brings an computational efficiency improvement for the domaindecomposition based parallelization.

\section{ACKNOWLEDGEMENTS}

The research leading to these results has received funding from the European Union's Horizon 2020 research and innovation programme under the ENERXICO project, grant agreement No. 828947. This study was also partially funded by the SEISCOPE consortium (http:// seiscope2.osug.fr), sponsored by AKERBP, CGG, CHEVRON, EQUINOR, EXXON-MOBIL, JGI, PETROBRAS, SCHLUMBERGER, SHELL, SINOPEC, SISPROBE and TOTAL. This study was granted access to the HPC resources of CIMENT infrastructure (https:// ciment.ujf-grenoble.fr), Cray Marketing Partner Network (https : //partners.cray.com) and CINES/IDRIS/TGCC under the allocation 046091 made by GENCI. 


\section{D acoustic-(visco)elastic coupled modeling}

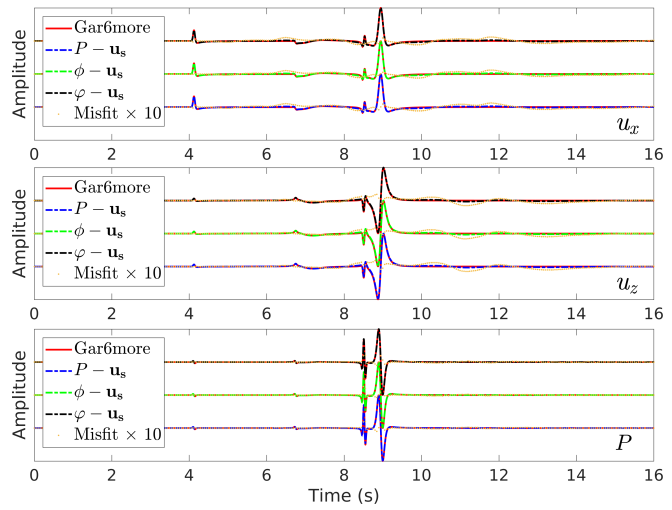

(a)

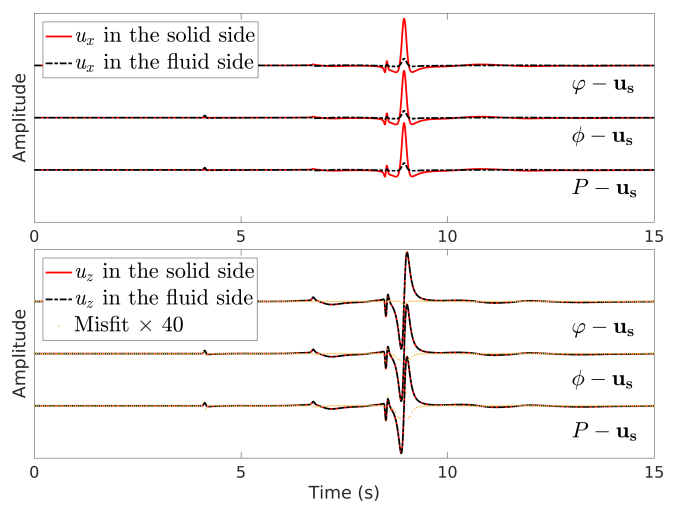

(b)

Figure 3: Validation for the modeling results of 2D isotropic bilayered model. (a) Benchmark against the semi-analytical solution (Gar6more2D (Diaz and Ezziani 2008), (b) continuity check at the interface. Receiver is located on the seabed with an offset of $12.6 \mathrm{~km}$.
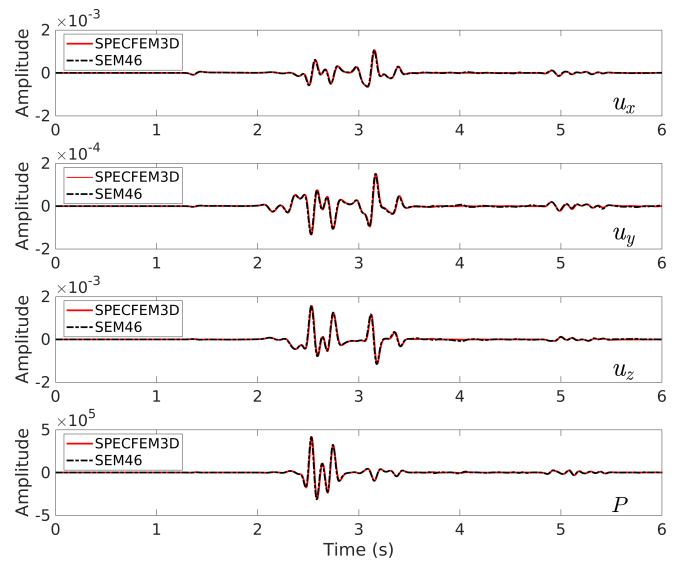

(a)
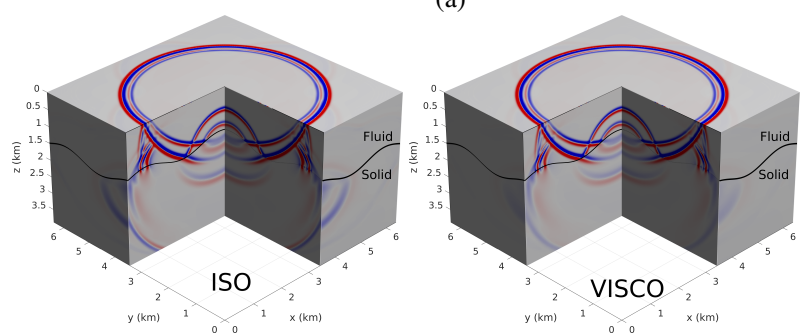
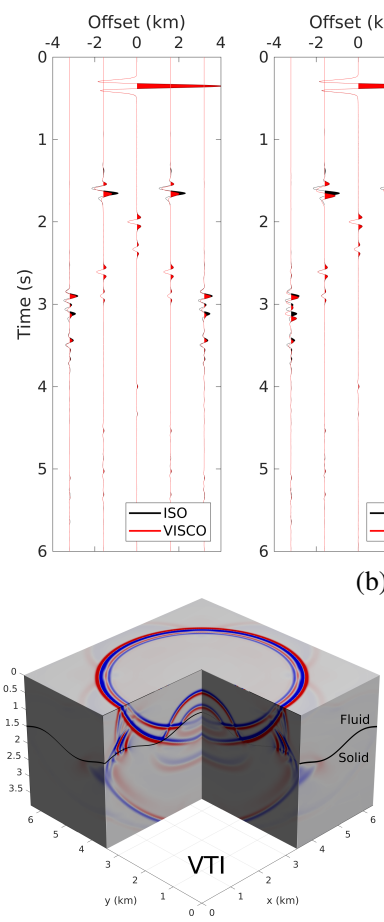

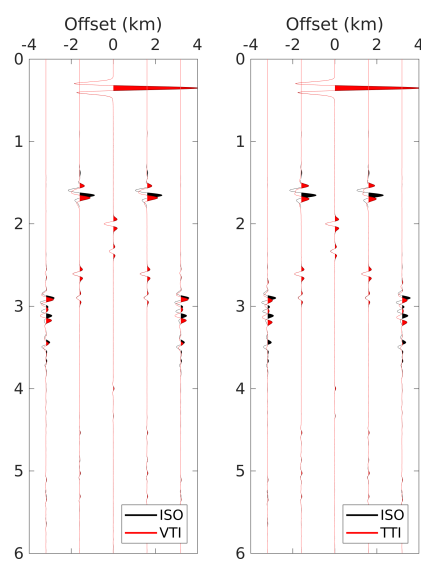

(b)

(c)

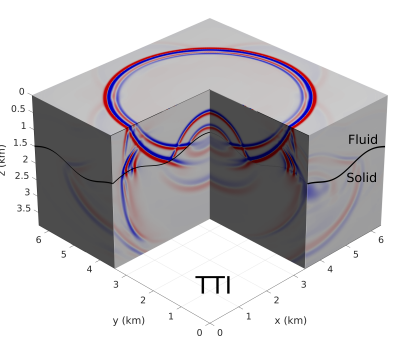

Figure 4: Modeling results of 3D bilayered models. (a) Seismogram comparison with results of SPECFEM3D (Peter et al. 2011) in the isotropic case, where the receiver is located on the seabed with the $x$ - and $y$ - offset of $2.8 \mathrm{~km}$ and $0.1 \mathrm{~km}$. Panels (b) and (c) are comparisons of pressure records and vertical displacement snapshots in the isotropic, viscous-isotropic, VTI and TTI cases, respectively.

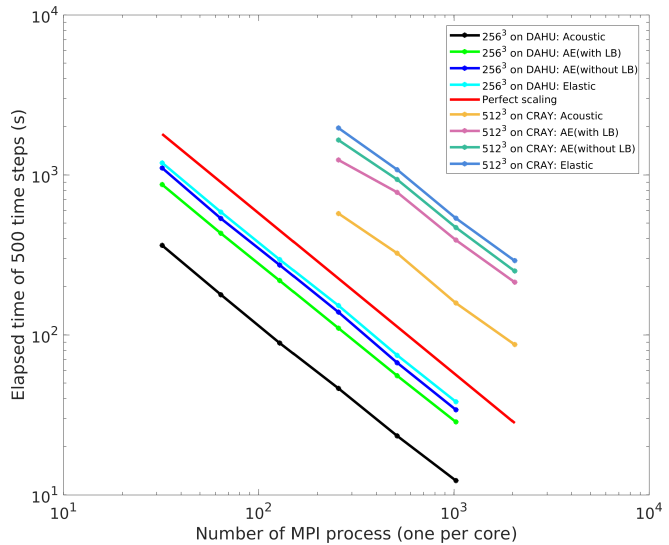

(a)

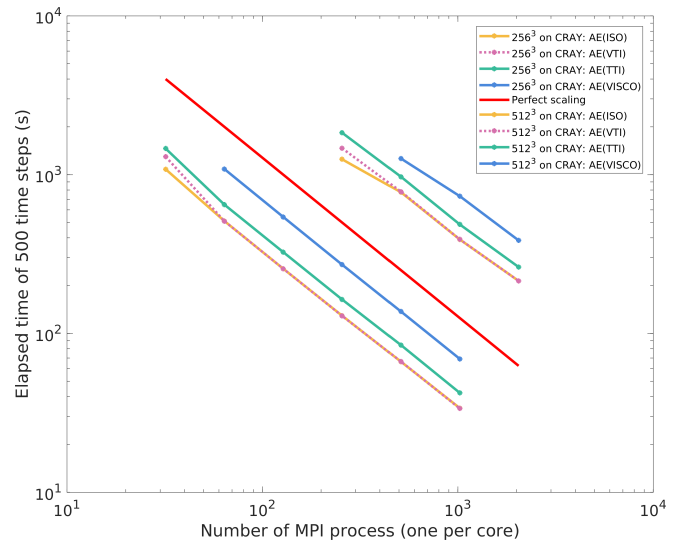

(b)

Figure 5: Scaling tests on two Intel-based clusters with $p^{4}$ elements: DAHU at Univ. Grenoble Alpes and SWAN plateform from CRAY. Panel (a) illustrates the parallel efficiency for full acoustic, full elastic, fluid-solid coupled without and with load-balance optimization, for square $256^{3}$ and $512^{3}$ element meshes. Panel (b) shows the parallel efficiency on CRAY machine for fluid-solid coupled modeling in the isotropic, viscous-isotropic, VTI and TTI cases. 